\title{
Antidiabetic Effect of Aqueous Seed and Endocarp Extracts of Swietenia Macrophylla King Combined with Glibenclamide in Rats
}

\author{
Hanan Kumar Gopalan, Nur Hayati Jaafar Marican*, Rahim Md Noah, Muhammad Rohani Jais and \\ Nor Shahirah Ahmad
}

Department of Biomedical Sciences, Institute of Medical Science Technology, University Kuala Lumpur, 43000 Kajang, Selangor Darul Ehsan, Malaysia; E-mail: hayyati.marican@gmail.com

Plants and plant products with antihyperglycemic capacity are often combined with the oral hypoglycemic drug for diabetes therapy. The plant-drug interaction may affect the pharmacology and toxicology of either component leading to a number of categorizing effects. This study investigated the antidiabetic effects of Swietenia's seed and endocarp aqueous extract in streptozotocin (STZ)-induced diabetic rats and to assess the possible plant-drug interactions with glibenclamide. The experimental groups were rendered diabetic by chemical combination of STZ (65 mg/kg bwt, i.v.) and NAD (230 mg/kg bwt, i.p.) in adult rats. Diabetic rats were orally force-fed with glibenclamide $(5 \mathrm{mg} / \mathrm{kg} \mathrm{bwt})$, extract $(250 \mathrm{mg} / \mathrm{kg} \mathrm{bwt})$ and a combination of full strength extract with half the glibenclamide dosage, daily for three weeks. Body weight (g) and FBG levels $(\mathrm{mg} / \mathrm{dl})$ were determined at treatment intervals of $0,7,14$ and 21 . Antidiabetic capacity of the aqueous plant extract $(P<0.001)$ were confirmed in the extract treatment group. Significant antidiabetic results were found in combined treatment of plant extract-drug group demonstrated by FBG reduction $(P<0.001)$ and body weight increment $(P<0.05)$ thus signifies the existence of potentiation effect. Findings implies that Swietenia macrophylla King seed and endocarp aqueous extract exhibits synergistic effect against diabetes and should serve as supplementation with a reduction in the dose of glibenclamide to warrant efficacy and safety.

Keywords: Swietenia macrophylla King, Interaction, Aqueous Extract, Antidiabetic, Synergistic. 